\title{
Sensitivity of a "dispersed-speckles" piston sensor for multi-aperture interferometers and hypertelescopes
}

\author{
V. Borkowski ${ }^{1,2}$, A. Labeyrie ${ }^{1}$, F. Martinache $^{1}$, and D. Peterson ${ }^{3}$ \\ ${ }^{1}$ Collège de France \& Laboratoire d'Interférométrie Stellaire et Exo-planétaire LISE-Observatoire de Haute-Provence - CNRS, \\ 04870 Saint-Michel l'Observatoire, France \\ e-mail: borkowski@obs-hp.fr \\ 2 Alcatel Space Industries, 100 bd du Midi, BP 99, 06156 Cannes la Bocca Cedex, France \\ 3 Department of Physics and Astronomy, Stony Brook University, Stony Brook, NY 11794-3800, USA
}

Received 13 February 2004 / Accepted 2 September 2004

\begin{abstract}
In multi-aperture interferometers and hypertelescopes, the piston errors can be determined from multi-spectral images of the speckle pattern using a 3-dimensional Fourier transform. We study the limiting magnitude of the method in the case of non-redundant apertures using analytical derivations and numerical simulations. We show specifically for the case of sub- $r_{0}$ apertures that a few photons per aperture within the full spectral bandwidth will suffice to measure piston errors. The method's sensitivity is thus comparable to that of the Shack-Hartmann and other methods used with monolithic apertures.
\end{abstract}

Key words. turbulence - techniques: interferometric - atmospheric effects

\section{Introduction}

The image enhancement achieved by adaptive optics in large telescopes requires mapping the wavefront bumpiness, i.e. measuring piston errors at many points across the aperture. The established methods for achieving such wavefront sensing, mainly the Shack-Hartmann and Curvature methods, rely upon the continuity of the wavefront. However, there is no such continuity in dilute multi-aperture interferometers. Their direct-imaging forms, called Fizeau interferometers, and "hypertelescopes" if there is a densified exit-pupil (Labeyrie 1996), therefore require different methods of piston sensing. A method for solving this more general problem was proposed by Labeyrie (Labeyrie 2002).

It extends to more than two apertures the group-delay or "dispersed fringes" measurement method used since Michelson and Pease (1921), Koechlin et al. (1996), and which now provides pairwise piston measurements for up to six apertures at the NPOI. Here, we calculate the limiting magnitude of this "dispersed speckles" method and verify with numerical simulations. In Sect. 2 we recall the principle of the algorithm. We briefly discuss the effect of redundancy, which requires refined procedures to discriminate the piston values. In Sect. 3, we discuss the sensitivity performance of the method, using numerical simulations with photon noise and analytical derivations. In Sect. 4, we discuss the comparison with other algorithms which have been proposed. In Sect. 5, we discuss the future prospects for this method. A separate article (Borkowski et al., in preparation) describes a prototype piston sensor instrument and its laboratory performance.

\section{Principle of the "dispersed speckles" algorithm}

As briefly described earlier (Labeyrie 2002; Borkowski et al. 2002) we consider the image of an unresolved guide star, formed with multiple apertures in the presence of piston errors. The image typically shows speckles, each having a spectrum which is itself speckled. The method extracts the information on piston errors which is contained in the spatio-spectral speckles.

The aperture may be diluted, and possibly highly diluted like in a long-baseline multi-element Fizeau interferometer, in which case it can be re-arrranged to provide a densified exit pupil as in a hypertelescope. The aperture can also be compact and highly redundant like in the Keck telescopes. Although this article deals mostly with non-redundant examples, much of it is applicable to redundant apertures, as discussed in more detail by (Martinache 2004). We ignore here the wavefront errors within each sub-aperture, i.e. tip-tilt, defocus and higher-order aberrations, since they can be analyzed efficiently with established sensing methods, and be corrected adaptively at each aperture separately. The issue is to remove the remaining piston offsets.

The speckled image is detected and recorded at a series of wavelengths simultaneously, using a spectro-imaging instrument. As described in Borkowski et al. (in preparation), a small spectrograph with a mosaic of optical wedges near its grating can do it by "exploding" the spectrum into a series of parallel spectra, each formed by one resel (resolution element) of the image. Unlike the usual spectrographs, the slit is replaced by a highly de-magnified pupil, and the image is highly magnified in the grating plane. Thus, there is typically one image speckle 

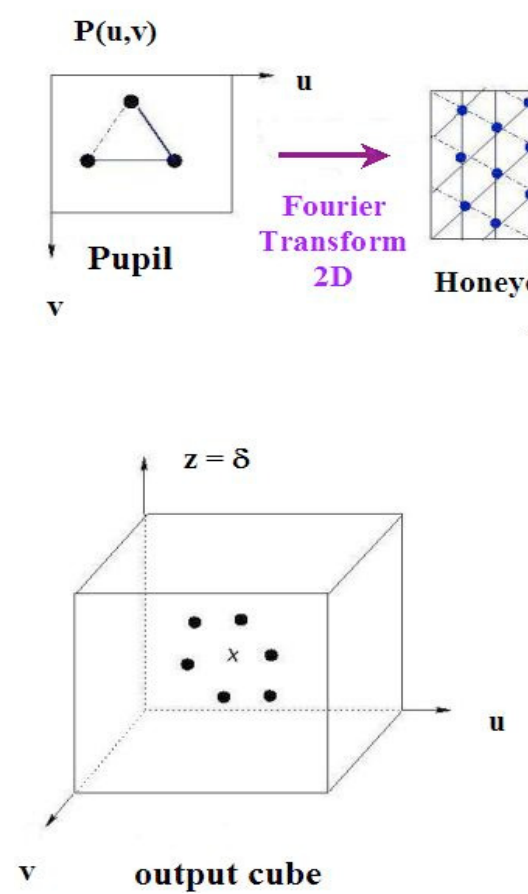
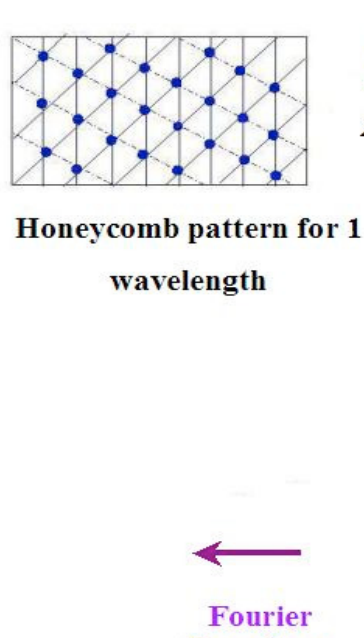
Transform 3D

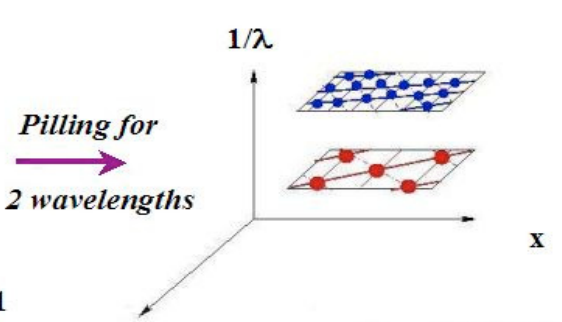

$\mathbf{y}$ several
Pilling for wavenumbers made linear $\sigma=1 / \lambda$

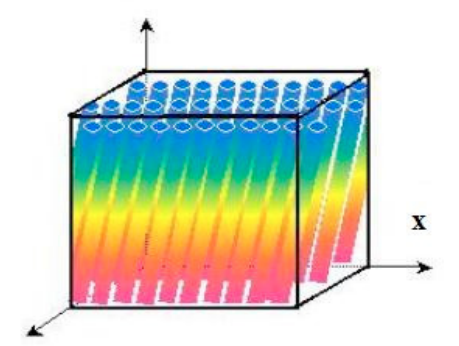

$\mathbf{y}$

\section{Dispersed image cube}

Fig. 1. Description of the algorithm. The example uses a triple aperture for a simplified ("honeycomb-like") 3-dimensional structure of dispersed speckles. Clockwise from top-left: A- aperture ; B- its Fourier transform, in intensity form, which is the recorded star image, in blue light. C- layering of a blue and red image, before re-scaling; D- input cube with all wavelength layers, re-scaled. With 3 apertures, it is a honeycomb pattern, tilted in response to the piston values. With more apertures, it has 3-dimensional speckles. E- output cube, calculated as a 3-dimensional Fourier transform of D. Its active columns contain "signal dots" at heights proportional to the piston value in the corresponding baseline. The six dots here obtained with 3 apertures are in a plane tilted like the piston value map.

received onto each wedge and providing a distinct spectrum. In the computer the spectra are rearranged as a 3-dimensional "dispersed speckles" image, interpolated to be linear in inverse wavelength. The monochromatic patterns are then layered into a "data cube" representing the dispersed speckle pattern (Fig. 1). Its 3-dimensional Fourier transform is then calculated to obtain an "output cube".

For each pair of apertures, defining a baseline, the output cube has an "active" column containing a "signal dot". Its position along the column is a measure of the piston difference among the two apertures. There are several dots in columns corresponding to redundant baselines. The positions of the "active" columns in the output cube match those of the peaks in the pupil's autocorrelation and the 3-dimensional Fourier transformation can be accelerated by calculating only these columns.

\subsection{The array's centro-symmetry and redundancy}

Martinache (2004) has shown that the data cube is the 3-dimensional energy spectrum of the unknown piston map, i.e. the square modulus of its 3-dimensional Fourier transform, if this map is defined as a binary function of both aperture coordinates and the piston value, with zeros and ones defining locally the presence of an aperture at all positions and possible piston values. He thus concludes that the output cube is a 3-dimensional autocorrelation function of the piston map. Both have real and positive values. These results show that the general problem of reconstructing the piston map is a problem of inverting the 3-dimensional autocorrelation. It has an exact, easily obtained at high illumination levels, if the aperture is non-redundant. There is however in all cases a spurious solution, related to the true solution through a 3-dimensional centro-symmetry. The true solution can be discriminated if the known aperture shape has no center of symmetry, like in the examples below, where an odd number of apertures are arrayed along a ring, with sometimes also one aperture at the center.

The centro-symmetry of a redundant array can easily be destroyed by removing or adding one aperture. A redundant array with an odd number of apertures can be centro-symmetrical only if it has a sub-aperture in the center. Solving for piston values in the redundant case proved possible when the aperture is not perfectly symmetrical using a 3-dimensional Fienup routine and more direct methods may prove feasible (Martinache 2004).

\subsection{The effect of photon noise}

At low illumination levels providing few photon events, the high-illumination-level speckled data cube becomes a 3-dimensional cloud of Dirac dots, assuming a photon counting detector. As in ordinary speckled images recorded with such detectors at low levels, the random Poisson sampling of the speckles results in a "compounded Poissone" statistical distribution, classically known as the Bose-Einstein distribution (Dainty 1974).

In the output cube, now calculated as the 3-dimensional Fourier transform of the Dirac cloud, the high-illuminationlevel pattern of $N(N-1)$ discrete "signal dots" ( $N$ is the number 
of apertures), real, positive, and having a dark background (the background is fully dark in the 3-dimensional autocorrelation of the wavefront map, but the finite size of the data cube intermediate here causes sidelobes to appear when calculating its Fourier transform), is now contaminated with a speckled background.

The contributing complex amplitudes being randomly phased, the average modulus of this background is $\sqrt{P}$, if $P$ is the number of photons in the Dirac cloud. The modulus of the central speckle, corresponding to the central dot of the high level pattern (i.e. with an infinite number of photons), which is the 3-dimensional auto-correlation of the piston map, is $P$ since it receives phased contributions. The modulus of the "signal dots", being $N$ times fainter at high illumination levels, is therefore $P / N$. Their signal/background modulus contrast is therefore $(P / N) / \sqrt{P}=\sqrt{P} / N$, and the intensity contrast is $I_{\text {peak }} / I_{\text {back }}=P / N^{2}$. The detection of a given signal dot with appreciable confidence requires that this contrast be significantly higher than 1, a condition which may be written $P>N^{2}$.

Since however $N-1$ output columns contain a signal dot pertaining to a given piston value, fewer photons will be needed in practice if the information from these output columns can be combined, and the condition then becomes $P>N$. At least, one photon event must be recorded from each sub-aperture, within the full bandwidth.

One way of achieving such a combination involves multiple correlations, as illustrated in Fig. 2. The computational burden grows faster than the $(N-1)$ th power of the number of piston steps. We have not yet attempted to include this correlation analysis in our simulations, and the results presented here are therefore likely to be pessimistic in terms of the limiting sensitivity and magnitude. An alternate method, the 3-dimensional Fienup algorithm used by Martinache (2004), does provide a global solution where the minimal number of photons is approximately $320 \times N$. It may be that other, more sensitive, methods will be found and will better approach the $N$ photon limit mentioned above.

Since a star of given magnitude provides a number of photons $P$ proportional to $N$, the limiting magnitude in this regime would not depend on $N$. The condition $P>N$, is similar to the limitation of a conventional Shack-Hartmann sensor using a lens array of $N$ elements, if the interferometer's sub-apertures are assumed to match $r_{0}$. Thus, with "dispersed-speckles" piston sensing, an $\mathrm{N}$-aperture diluted interferometric array or hypertelescope can be phased with adaptive optics and be expected to reach the same limiting magnitude for the guide star as a monolithic adaptive telescope having the same collecting area and $N$ actuators.

Applications such as coronagraphy require a phasing accuracy much better than $\pi / 2$. This takes more photons than calculated above. Indeed, the background speckles in the output columns not only affect the detection of the signal dots, when their level is comparable, but also affect slightly the position of their photocenter along the column, even when the signal dot is much more intense than the neighbouring speckles.

This effect of "dot pushing" along a column of the output cube can be crudely modelled locally by adding complex amplitude distributions typical of a signal dot and of a fainter

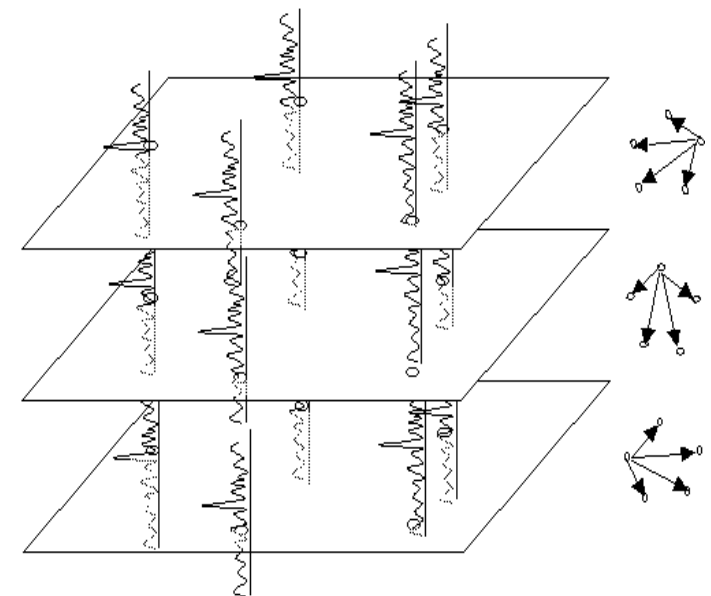

Fig. 2. Statistical reconstruction of piston values from the output cube, here shown in the case of a 5-aperture circle. $N$ subsets from the set of output columns (only 3 of 5 are shown here), each related to a given "starting" aperture, are extracted from the cube and repositioned so that identical "target" apertures (indicated by arrows in the sketches at right) are aligned vertically in separate layers. The set of signal dots in each layer is, in principle, globally translated vertically according to the piston value of the "starting" aperture. This error can be determined by intercorrelating the layers, using a multiple correlation of order $(N-1)$ applied to the vertical translations along the columns.

speckle peak located close to it, within less than a speckle diameter. Near both peaks, their moduli profiles along the column can be approximated by a quadratic function of the $\Delta \delta$ position relative to the true position of the signal dot. These can be expressed respectively as $\left(1-(\Delta \delta)^{2}\right) / 2 R$ and $\varepsilon\left(1-(\Delta(\delta-a))^{2}\right) / 2 R$, a similar approximation of the closest speckle, shifted by $a$ along the column and with relative modulus $\varepsilon . R$ is the curvature radius of the peaks, of the order of the speckle size. Depending on the phase difference, the complex addition tends to displace the signal dot towards the contaminating speckle or away from it. It has a neutral effect in the case of quadrature phase.

The displacement in the column direction is of the order of $\varepsilon a$ Pending refined calculations, $a$ may be taken as typically equal to the speckle size, thus indicating that the signal dot's position error along the column is, in units of the speckle size, of the order of $\Delta D o t=\varepsilon=N / \sqrt{P}$. Since the speckle unit corresponds to the high-illumination-level vertical resolution of piston measurements, which is $\lambda^{2} / \Delta \lambda$, the piston measurement accuracy in length units is $\epsilon_{\mathrm{pos}}=\left(\lambda^{2} / \Delta \lambda\right) N / \sqrt{P}$.

If measurements from the $N$ signal dots carrying information on a given piston are combined, for example as shown in Fig. 2 the error drops as $\sqrt{N}$, thus becoming $\epsilon_{(\mathrm{pos}) 2}=$ $\left(\lambda^{2} / \Delta \lambda\right)(\sqrt{N} / \sqrt{P})$. If the array is expanded by adding apertures, the star magnitude being fixed, then $P=k N$ and $\epsilon_{(\mathrm{pos}) 2}=$ $\left(\lambda^{2} / \Delta \lambda\right) \sqrt{k}$ remains constant. If instead the sub-aperture sizes are changed along with their number to keep a constant collecting area, then $P$ is constant on a given star and $\epsilon_{(\mathrm{pos}) 2}$ varies as $\sqrt{N}$. Figure 4 shows that these expressions are consistent with the simulation results in the region where the $I_{\text {peak }} / I_{\text {back }}$ contrast is moderate, less than 100 , which is the region of practical interest. At the higher contrast values, the analytical 
expression overestimates markedly the contrast. A good reason for this discrepancy is that the analytical derivation ignores the presence of side-lobes associated with each signal dot even at high photon levels, a consequence of the data cube's finite size. The contamination can be attenuated, if needed when dealing with many apertures, by apodizing the data cube. Its two image dimensions are naturally apodized if the image envelope is smaller than the pixel array.

The relations found between the accuracy of piston measurements, the number of apertures and the number of photons collected do not depend on the range of piston values. A larger range obviously requires a denser sampling of the input cube, along the wavenumber axis, so as to resolve highly tilted fringes. More spectral channels are therefore needed in the instrument, within a given spectral range, but our equations indicate that the accuracy of piston measurements remains constant if the total number of photons detected within this range is kept constant.

\subsection{The simulation algorithm}

We have simulated the formation of the spectro-images or data cube by calculating a series of 2-dimensional Fourier transforms from the complex amplitude pattern in the aperture, where a single point is assigned to each sub-aperture. Incremental wavenumbers $\sigma$ are used for each Fourier transform. Instead of doing the chromatic re-scaling post-detection, as will be done when using the real instrument, the simulation does it directly when calculating the image, by modifying the classical phase expression $2 \pi \sigma(u x+v y+\Delta(u, v))$ in the formulation of the Fourier transform. Instead, the chromatically re-scaled series of Fourier transforms are here expressed as:

$I(x, y, \sigma)=\left|\iint P u p(u, v) \mathrm{e}^{(-2 i \pi(u x+v y+\sigma \Delta(u, v)))} \mathrm{d} u \mathrm{~d} v\right|^{2}$

where $I$ is the image intensity, $\operatorname{Pup}(u, v)$ describes the interferometer entrance pupil configuration, $\Delta(u, v)$ is the optical path difference resulting from static piston values and the atmospheric turbulence, $x$ and $y$ are the spatial frequencies in the Fourier domain, $u=\frac{u^{\prime}}{\lambda f}, v=\frac{v^{\prime}}{\lambda f}, \sigma=\lambda^{-1}$ and $f$ (the focal length) is included in $\Delta$. The monochromatic images, thus re-scaled and calculated at incremental values of $\sigma$, are numerically stacked and Poisson sampled to generate the simulated "input cube" having limited photon count. Its 3-dimensional Fourier transform is then calculated to generate the output cube where signal dots may be searched in those columns which are located at the auto-correlation points of the aperture.

The 3-dimensional Fourier transform producing the output cube may then be expressed as:

$\left.\operatorname{output}(u, v, z)=\mid \iiint I(x, y, \sigma) \mathrm{e}^{-2 i \pi(u x+v y+\sigma z)} \mathrm{d} x \mathrm{~d} y \mathrm{~d} \sigma\right)\left.\right|^{2}$

The "output cube" is simply the 3 dimensional Fourier transform of the dispersed image cube (Fig. 1).

\section{Minimal photon count found from numerical simulations}

As discussed in Sect. 2.2, a photon-starved data cube generates a degraded output cube where a speckled background affects the visibility of the signal dots. Measuring the position of a signal dot along its column to derive the corresponding piston value first requires that the dot be correctly identified among the speckles. The confidence level improves with the peak/background contrast. If the dot is correctly identified, the accuracy of the piston measurement remains affected by the dot's deformation, induced by the neighbouring speckles.

Our simulation code calculates the peak/background contrast of signal dots and then finds, from a series of exposures made with a single frozen piston map, the dispersion of the piston measurements, indicative of the accuracy reachable. At this stage however, no attempt was made to exploit the piston information redundancy mentioned in Sect. 2.2, and the sensitivity estimates given here are therefore pessimistic. Trial and error indicated the photon count needed to achieve piston measurements accurate enough for: 1) diffraction-limited imaging, according to Rayleigh's $\lambda / 4$ classical tolerance (i.e. 0.1 to $0.2 \mu \mathrm{m}$ at visible wavelengths); and 2) coronagraphic imaging with $\lambda / 100$ tolerance (i.e. 4 to $8 \mathrm{~nm}$ at visible wavelengths).

We present here results for ring arrays having 3 to 10 apertures. In cases with an even number $N$ of apertures we locate $N-1$ apertures on a ring and one at the center to make the configuration non-redundant. All the simulations are made with 64 wavelengths. As expected, the number of spectral channels, adjacent within the $400-800 \mathrm{~nm}$ visible bandwidth utilized, influences the maximal piston value but not the minimal photon count. The photon counts are specified per data cube.

\subsection{Intensity profiles in active columns}

We have looked at the column profiles in order to determine the minimal photon count needed to detect the signal dot. Typical column profiles are shown for ring pupils with six apertures (Fig. 3). As expected, the column background, normalized to the intensity of the signal peak, is dark at high photon level and speckled at lower levels.

Figure 4 indicates the evolution of the peak's contrast $I_{\text {peak }} / I_{\text {back }}$ averaged on all the $N(N-1)$ active columns versus the number of apertures, at constant total photon count. With 6 apertures, 320 photons per cube or 54 photons per aperture are needed to recover piston values as can be seen on top of Fig. 3. The average $(S / N)$ is approximately 8. In Fig. 4, a signal/noise ratio $S / N$ higher than 4 in each column, provides a sufficient confidence level for distinguishing signal from noise. The ratio $I_{\text {peak }} / I_{\text {back }}$ varies from column to column but its average on all the columns has to be larger than 5. With 10 apertures, a total of 960 photons, thus 96 photons per aperture (at the bottom of Fig. 3), are needed. The ratio $I_{\text {peak }} / I_{\text {back }}$, which is approximately equal to 8 , is averaged on all the active columns within the data cube, thus, for some of them, less than 960 photons will suffice to determine the piston values. The minimal number of photons providing detectable signal dots in the output columns, and therefore measurable piston values, in each 

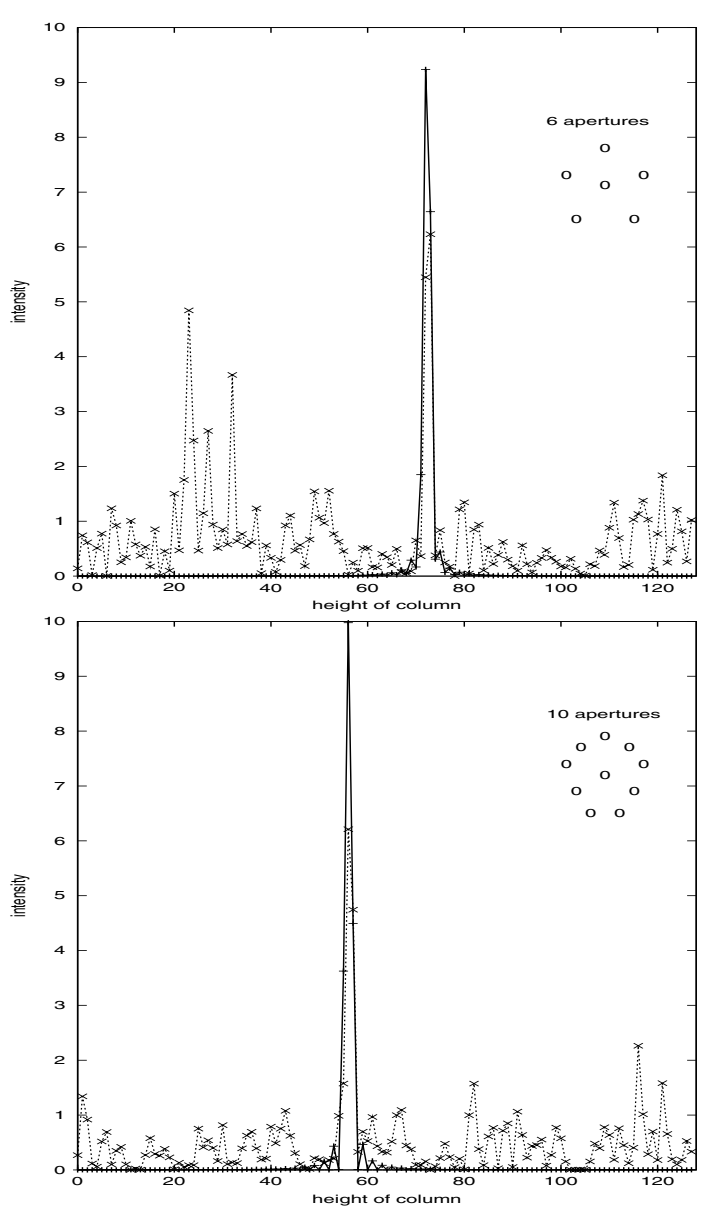

Fig. 3. Intensity along one active column with no noise (solid lines) and with one realization of photon noise (dotted lines) Top: with 320 photons per cube for six apertures arranged as shown. Bottom: with 1600 photons per cube for ten apertures arranged as shown.

aperture configuration, are summarized in Fig. 5 (curve labelled minimum) and in Table 1.

\subsection{Accuracy of piston measurements deduced from the simulations}

Our estimates for the accuracy of the piston measurements are summarized in Fig. 5 and in Table 1. With 6 apertures, precisions better than $\lambda / 100$ are achieved with $1.6 \times 10^{5}$ photons per data cube or $2.67 \times 10^{4}$ per aperture. With 10 aperture, precisions better than $\lambda / 100$ are reached with $3.2 \times 10^{5}$ photons per data cube or $3.2 \times 10^{4}$ per aperture. With 6 apertures, precisions better than $\lambda / 4$ are achieved with $1.6 \times 10^{4}$ photons per data cube or 2667 per aperture. In the case of an interferometer with 10 apertures $3.2 \times 10^{4}$ photons per data cube or 3200 per aperture are needed so as to reach $\lambda / 4$ accuracy.

\subsection{Limiting stellar magnitude}

We used the numbers of photons indicated by the simulations to calculate the stellar magnitudes reachable. The limiting stellar magnitude $m_{v}$ for guide stars, again in the same

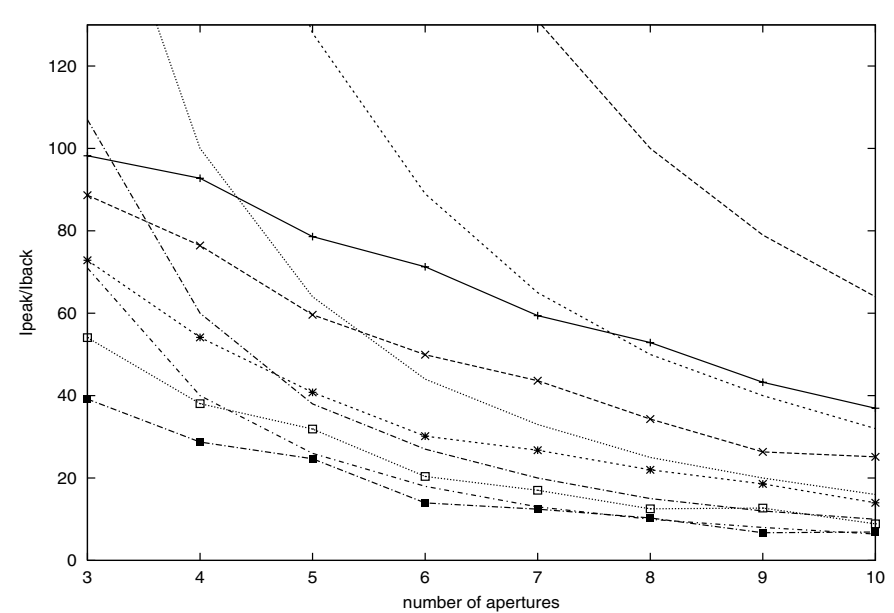

Fig. 4. Contrast of the signal dots with 3 to 10 apertures. Curves with line points show simulations with, from top to bottom, 6400 photons, 3200 photons, 1600 photons, 960 photons and 640 photons per cube. From top right to bottom, curves without line points are based on theory, showing cases with 6400 photons, 3200 photons, 1600 photons and 960 photons per cube.

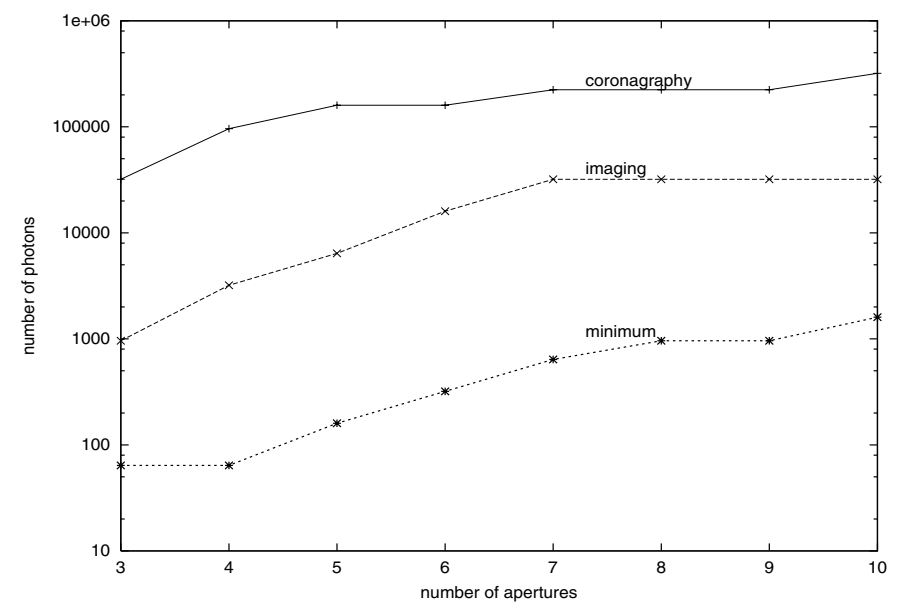

Fig. 5. From top to bottom: evolution of the number of photon needed in order to do coronagraphy, imaging and recover signal from noise with 3 to 10 apertures.

pessimistic case, is calculated with Muller \& Buffington's expression (Muller \& Buffington 1974).

$m_{v}=2.5 \log (734 \Delta \lambda / B)$

where $\Delta \lambda$ is the total optical bandwidth in $\ddot{\mathrm{A}}$ and $B$ is the photon flux per $\mathrm{cm}^{2}$.

$B=4 p / \pi d^{2} \tau \eta N$

where $p$ is the number of photons, $d$ is the diameter of subapertures in $\mathrm{cm}, \tau$ is the seeing lifetime, $\eta$ is the overall quantum efficiency. For the numerical calculations we use $\tau=0.02 \mathrm{~s}$ and $\eta=0.1$. We have chosen the third dimension of our input cube as:

$\lambda^{-1}=1.25+0.02 n$

with $n=0$ to 63 for 64 wavelengths, thus $\Delta \lambda \simeq 4000 \AA$. 
Table 1. Evolution of the number of photons, limiting magnitudes and Strehl ratio in function of the number of apertures and in function of the considered mode. Imaging and coronagraphic modes require respectively precision of $\lambda / 4$ and $\lambda / 100$. From left to right: 1 - number of apertures; 2- minimum, imaging or coronagraphic modes; 3- number of photons needed to achieve the required precision; 4- limiting stellar magnitude for an optical bandwidth of $\Delta \lambda=4000$ Åand for a constant aperture diameter of $8 \mathrm{~m}$; 5- limiting stellar magnitude for a collecting surface of $150 \mathrm{~m}^{2}\left(\pi *(8 / 2)^{2} * 3\right)$; 6- Strehl ratio.

\begin{tabular}{|c|c|c|c|c|c|}
\hline $\begin{array}{l}\text { Number } \\
\text { of } \\
\text { apertures }\end{array}$ & Mode & $\begin{array}{c}\text { Number } \\
\text { of photons } \\
\text { needed } \\
\text { per cube }\end{array}$ & \multicolumn{2}{|c|}{$d=8 \mathrm{~m} S_{\text {coll }}=150 \mathrm{~m}^{2}$} & $\begin{array}{l}\text { Strehl } \\
\text { (in \%) }\end{array}$ \\
\hline \multirow{3}{*}{3} & Minimum & 64 & 20.4 & 20.4 & 83 \\
\hline & Imaging & 960 & 17.4 & 17.4 & 94 \\
\hline & Coronagraphy & 32000 & 13.6 & 13.6 & 100 \\
\hline \multirow{3}{*}{4} & Minimum & 64 & 20.7 & 20.7 & 52 \\
\hline & Imaging & 3200 & 16.4 & 16.1 & 95 \\
\hline & Coronagraphy & 96000 & 12.7 & 12.4 & 98 \\
\hline \multirow{3}{*}{5} & Minimum & 160 & 19.9 & 19.6 & 63 \\
\hline & Imaging & 6400 & 15.9 & 15.4 & 94 \\
\hline & Coronagraphy & 160000 & 12.4 & 11.7 & 97 \\
\hline \multirow{3}{*}{6} & Minimum & 320 & 19.4 & 18.6 & 63 \\
\hline & imaging & 16000 & 15.1 & 14.4 & 90 \\
\hline & Coronagraphy & 160000 & 12.6 & 11.9 & 97 \\
\hline \multirow{3}{*}{7} & Minimum & 640 & 18.8 & 17.6 & 64 \\
\hline & imaging & 32000 & 14.5 & 13.6 & 93 \\
\hline & Coronagraphy & 224000 & 12.4 & 11.5 & 97 \\
\hline \multirow{3}{*}{8} & Minimum & 960 & 18.5 & 17.4 & 45 \\
\hline & Imaging & 32000 & 14.7 & 13.6 & 93 \\
\hline & Coronagraphy & 224000 & 12.6 & 11.5 & 96 \\
\hline \multirow{3}{*}{9} & Minimum & 960 & 18.6 & 17.4 & 47 \\
\hline & Imaging & 32000 & 14.8 & 13.6 & 84 \\
\hline & Coronagraphy & 224000 & 12.7 & 11.5 & 98 \\
\hline \multirow{3}{*}{10} & Minimum & 1600 & 18.2 & 16.9 & 63 \\
\hline & Imaging & 32000 & 14.8 & 13.6 & 81 \\
\hline & Coronagraphy & 320000 & 12.41 & 11.1 & 95 \\
\hline
\end{tabular}

The results are summarized in Table 1 and in Fig. 6, for a constant sub-aperture size of $8 \mathrm{~m}$ or for a constant collecting interferometer area. The varying sub-aperture size in the latter case, in the presence of adaptive optics within the subapertures, changes the optimal exposure duration. The simulation curves however use fixed exposures. Basically, more apertures require more photons. Figure 6 shows, for a given number of apertures, a gap of 2 mag (particularly for 10 apertures) between imaging and coronagraphy curves. Indeed, if the diameter of sub-apertures $d$ is constant, with 10 apertures, $S_{\text {coll }}$ (the collecting interferometer area) increases significantly $\left(5 \times 10^{6} \mathrm{~cm}^{2}\right)$ compared to the case where $d$ varies in order to keep $S_{\text {coll }}$ constant.

\subsection{Signal attenuation}

In order to have an idea of the performance attainable, we have calculated the signal attenuation obtained with the accuracy levels found above for the coronagraphic, imaging and

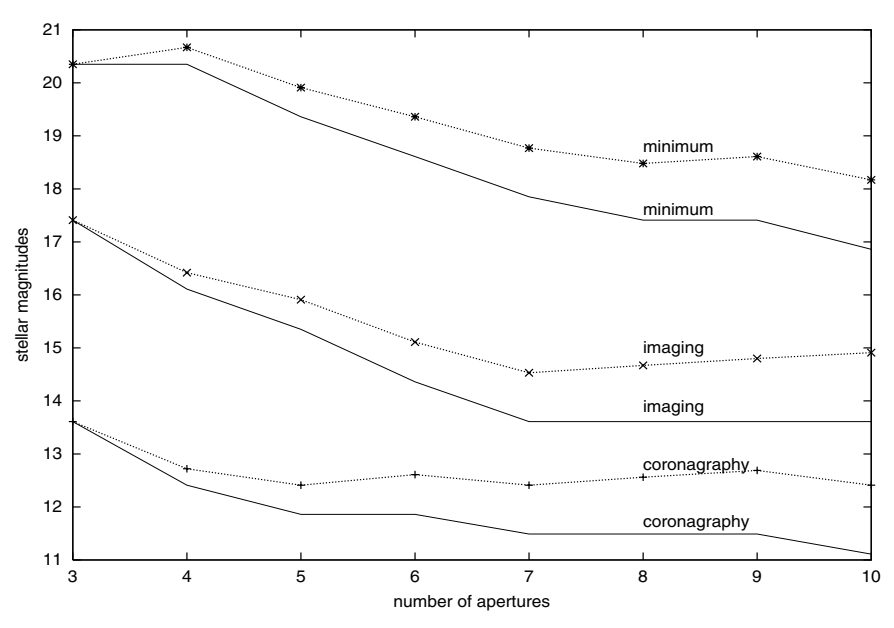

Fig. 6. From top to bottom: evolution of the limiting stellar magnitudes for coronagraphy, for imaging and for minimal signal detection, with 3 to 10 apertures. Solid curves correspond to interferometers with a constant collecting area of $150 \mathrm{~m}^{2}$ and dotted lines to a constant $8 \mathrm{~m}$ aperture size. Exposures last $0.02 \mathrm{~s}$ and the global quantum efficiency is $10 \%$.

minimum modes (see Table 1). Considering four $8 \mathrm{~m}$ telescopes, organized as the 4 UTs (Unit Telescope) of the Very Large Telescope Interferometer (VLT), the signal attenuation is approximately equal to $98 \%, 95 \%$ and $52 \%$ respectively for coronagraphy, imaging and recover signal from noise, the number of photons per cube being respectively $9.6 \times 10^{4}, 3.2 \times 10^{3}$ and 100, and the stellar magnitude 12.72, 16.42 and 19.91 .

Pending more complete simulations of our method, it can be remarked that the signal attenuation is not much degraded when the number of apertures increases if the total number of photons remains higher than $3 \times 10^{5}$ photons per data cube. With 10 apertures and $3 \times 10^{5}$ per cube, the signal attenuation is above $70 \%$.

\section{Discussion and future prospects}

The method allows phasing apertures efficiently with a wave sensor instrument which can also serve as the science camera. The case of redundant apertures, discussed elsewhere, is of possible interest for compact mosaic mirrors such as those of the Keck and Grantecan telescopes, as well as their Extremely Large Telescope successors. These have required edge sensors to position the mirror segments, but they may become unnecessary with the dispersed-speckles method if guide stars are available.

The VIDA (VLTI Imaging with a Densified Array) mode of hypertelescope observing proposed for the VLT interferometer (Lardière et al. 2002) may also benefit from the global piston measurement achieved with the dispersed-speckles method, in spite of the small number of apertures. It consists of coupling the VLT telescopes using a densified pupil mode. When the global reduction algorithm, exploiting the redundant information in output columns, will be fully optimized and coded for simulations, it will be of interest to compare with other multiaperture piston sensing methods, such as those of (Pedretti 1999), where beams are combined triplet-wise hierachically 
(hereafter called method 1), pair-wise (method 2 ) or with 2dimensional Fourier transforms (method 3) for non redundant configurations. The signal attenuations were about $52 \%, 99 \%$ and $98 \%$ respectively for the 3 methods and for $3 \times 10^{7}$ photons, 8 wavelengths and 27 apertures. For $3 \times 10^{4}$ photons, these signal attenuations remain unchanged

On Earth the method may be applied for active or adaptive piston sensing. Output cubes, calculated from short exposures, can be co-added to gain sensitivity. The signal dot in each column moves in response to the phase shifts of seeing and the shadow pattern. The time-integrated dot is therefore widened but gives the average piston value, usable for active optics. If detectable, the short-exposure dot can also be exploited to activate fast actuators for adaptive piston correction.

For coronagraphs the correction is perhaps best with wave sensing achieved coarsely before the coronagraph and finely with an additional wave sensor probing the residual speckles after the coronagraph. The "dispersed speckles" method is applicable in both stages. One or preferably two stages of actuators provide the correction, preferably before and after the coronagraph (Codona \& Angel 2004, submitted; Labeyrie 2004, in preparation). These results are encouraging and justify laboratory testing.

\section{Conclusion}

Unlike the established wavefront sensing methods, such as the Shack-Hartmann, requiring continuous optical surfaces, the dispersed-speckles method is applicable to multi-aperture imaging interferometers of arbitrary dilution and particularly to those providing hypertelescope images, with a densified pupil. Theory and the preliminary simulations reported here are consistent and indicate that a high sensitivity, approaching that of Shack-Hartmann sensing, can be expected. Limiting magnitudes of the order of 23.7 appear attainable for the guide star in the case of the VIDA hypertelescope imager proposed for the VLTI if each sub-aperture is already corrected with conventional adaptive optics. This is also consistent with the simulation results of (Martinache 2004), using a different mathematical algorithm for data reduction. Both indicate that large numbers of sub-apertures can be used, and the method therefore seems applicable to the mosaic mirrors of Keck and various proposed successors, for which edge sensors may become unnecessary in the presence of adequate guide stars. The method is also of interest for the demanding needs of coronagraphy, with instruments such as the Exo-Earth Discoverer, a proposed space hypertelescope designed for detecting ExoEarths (Labeyrie 2002). A simple prototype of an instrument to test these principles has been built by some of us (AL, VB). The results will be reported on separately (Borkowski et al., in preparation).

Acknowledgements. The authors are grateful to Ettore Pedretti for helpful discussions. This paper is part of the Ph.D. Thesis of V. Borkowski.

\section{References}

Borkowski, V., Martinache, F., Peterson, D., \& Labeyrie, A. 2002, Proc. ESLAB 36 Symposium, Earth-Like Planets and Moons, ESTEC, Noodwijk, 514, 273

Codona, J. L., \& Angel, R. 2004, ApJ, submitted

Dainty J. C. 1974, MNRAS, 169, 631

Hartmann J. 1900, Z. Instrumenkd, 20, 47

Koechlin L., Lawson, P. R., Mourard, D., et al. 1996, Appl. Opt., 35, 3002

Labeyrie, A. 1996, A\&AS, 118, 517

Labeyrie, A., Borkowski, V., Martinache, F., et al. 2002, Proc. ESO Venice Conf., Beyond Conventional Adaptive Optics, 58, 109

Labeyrie, A. 2002, Proc. ESLAB 36th Symp., Earth-Like Planets and Moons, ESTEC, 245

Lardière, O., Dejonghe, J., Riaud, P., et al. 2002, ASP Conf. Ser., 266, 608

Lardière, O., Labeyrie, A., Mourard, D., et al. 2002, Poc. SPIE, 4838, 1018

Martinache, F. 2004, J. Opt. A: Pure Appl. Opt., 6, 216

Michelson, A. A., \& Pease, F. G. 1921, AJ, 53, 249

Muller, R. A., \& Buffington, A. 1974, J. Opt. Soc. Am., 64, 9

Pedretti, E., \& Labeyrie, A. 1999, A\&AS, 137, 543

Pedretti, E. 1999, ASP Conf. Ser., 194, 271

Roddier, F. 1988, Appl. Opt, 27, 1223

Shook P. V., \& Platt, B. C. 1971, J. Opt. Soc. Am., 61, 656 